Unfallchirurg 2015 · [Suppl 1]: 118:S43-S52

DOI 10.1007/s00113-015-0100-1

Online publiziert: 16. November 2015

(c) Springer-Verlag Berlin Heidelberg 2015

\author{
P. Gruber ${ }^{1,2} \cdot$ T. Bönii ${ }^{2,3}$ \\ ${ }^{1}$ Klinik für Neurologie, Universitätsspital Zürich, Zürich, Schweiz \\ 2 Institut für Evolutionäre Medizin, Universität Zürich, Zürich, Schweiz \\ ${ }^{3}$ Orthopädische Universitätsklinik Balgrist, Zürich, Schweiz
}

\title{
Ischias
}

\section{Von der Säftelehre zur Pathomorphologie}

\begin{abstract}
Obwohl heute das diskogene Nervenkompressionssyndrom zu einer der häufigsten Krankheiten überhaupt gehört, verhalfen erst Mixter u. Barr [1] in den 30er Jahren des vergangenen Jahrhunderts diesem Krankheitsbild zum Durchbruch und zu allgemeiner Anerkennung in der medizinischen Öffentlichkeit. In diesem Beitrag wird der ideengeschichtlich keineswegs geradlinige Weg vom unspezifischen, auch Gelenkschmerzen beinhaltenden "Ischias" der Antike zum noch heute gültigen pathologisch-anatomisch verifizierten Krankheitskonzept des radikulären Kompressionssyndroms verfolgt. Die durch den Bandscheibenvorfall verursachte Kompression neuraler Strukturen steht dabei im Zentrum der Darstellung, die folgerichtig mit der Entdeckung von Mixter und Barr endet. Die Untersuchung ist eine überarbeitete Version [2] und basiert auf Vorarbeiten [3] der Autoren.
\end{abstract}

\section{Rückenbeschwerden im Alten Ägypten}

Im berühmten in hieratisch geschriebenen Papyrus Edwin Smith, der eine altägyptische chirurgische Textsammlung mit 48 Fallbeispielen aus der Zeit des frühen Neuen Reichs (1550 v. Chr.) ist, findet sich unter Fall 48 eine leider nicht vollständig erhaltene Fallvignette mit Rückenbeschwerden [4]. Entsprechend der systematischen Fallpräsentation, wie sie typisch für den Edwin-Smith-Papyrus und für weitere altägyptische medizinische Lehrtexte ist, welche sich jeweils in einer
Überschrift, Untersuchung, Diagnose/ Prognose und Therapie gliedert, wird folgendes dargelegt: „... Wenn du (einen Mann) untersuchst (mit) einer Zerrung an einem Wirbel seines Rückens, dann musst du zu ihm sagen: ,Strecke doch deine Beine aus, die (jetzt) angezogen sind!' Dann streckt er sie aus, und er zieht sie sofort wieder an, weil es schwierig ist, dass er (es) ausführt, infolge des Wirbels seines Rückens, an dem er dann Schmerz empfinden wird. Dann musst du dazu sagen: einer mit einer Zerrung an einem Wirbel seines Rücken. Eine Krankheit, die ich behandeln werde...." [4].

Diese Fallbeschreibung gibt Zeugnis $a b$, dass bereits die altägyptischen Ärzte Rückenbeschwerden behandelt haben und möglicherweise den Ischiasdehungsschmerz als diagnostischen Test (mit Vorbehalt) verwendeten. Die anatomischen und pathophysiologischen Kenntnisse waren zu jener Zeit indes beschränkt.

\section{Rückenbeschwerden in der babylonischen Medizin}

Neben der altägyptischen Medizin finden sich auch im Zweistromland v. a. für das 1. Jahrtausend vor Chr. reichhaltige medizinische Dokumente, die auf Tontafeln festgehalten wurden. Es lassen sich in diesen Texten Hinweise finden, dass die babylonischen Ärzte durchaus mit Rückenbeschwerden vertraut waren und hierfür 3 verschiedene Begriffe verwendeten, die symptomatisch verschiedene Zustände bezeichneten [5]. Der folgende Text zeigt eindrücklich die Beschreibung der Schmerzen: „... Es sticht wie der Biss eines Wolfes, es schiesst ein wie der Sprung des Hund von Elam; (...) Es kommt und geht wie ein Wildtier kommt und geht; Geh weg, maskadu (eine Form von Rückenbeschwerden), bevor das Steinmesser und Rasiermesser von Gula (Gottheit) dich packt!..." [5].

Aufgrund der Beschreibung bleibt es aber schwierig zurückschließen, was die Ursache der jeweiligen Rückenschmerzen war.

\section{Hippokrates von Kos und die hippokratischen Schriften}

In der Antike haben sich sowohl Hippokrates von Kos (460-370 v. Chr.) als auch die sogenannten hippokratischen Autoren, da wohl der bedeutende hippokratische Textkorpus nicht nur aus einer Hand stammt, mit dem Symptomkomplex des Lenden-Gesäß-OberschenkelSchmerzes auseinander gesetzt und diesen als "Ischias“ bezeichnet. Der noch heute verwendete Begriff „Ischias“ leitet sich vom griechischen Wort ,ischion “ $a b$, welches neben der Hüfte auch das Gesäß, das Sitzbein, die Lenden sowie gelegentlich auch den gesamten Oberschenkel bezeichnet. Dementsprechend behandelt ein ganzes Kapitel der hippokratischen Schrift „Vorhersagen II“ (Kap. 41; Praedictiones II) den „Ischias“ [6]. Die antiken griechischen Ärzte haben nicht nur dieses Krankheitsbild nach den Symptomen, sondern auch nach dem Krankheitsverlauf zwischen dem „Ischias“ der jungen und alten Patienten unterschieden. Bei den jungen Patienten wurde von einem kurzen Krankheitsverlauf berichtet, der nach 6 Wochen abklingen sollte. Hingegen zeichnet sich der "Ischias" der älteren Patienten durch einen chronischen 

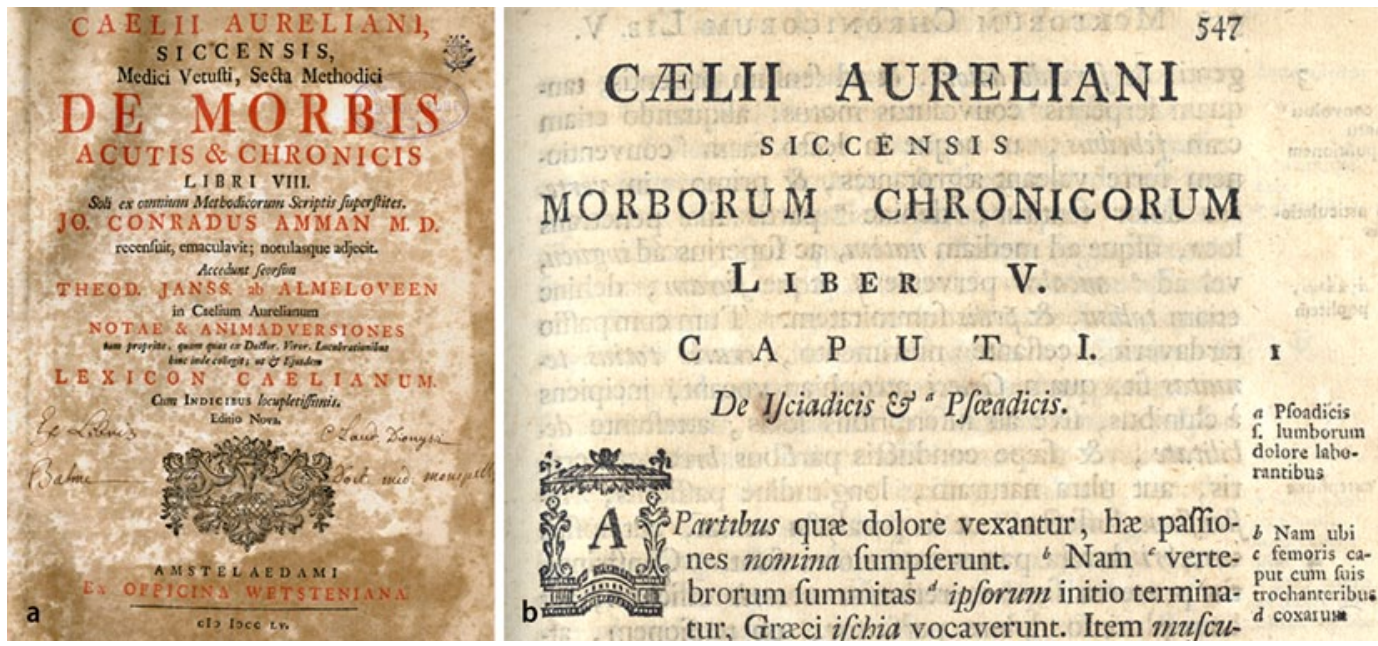

Abb. $1 \Delta$ a Titelblatt des von Caelius Aurelianus (5. Jhdt. nach Chr.) verfassten und vom Schaffhauser Arzt Johann Conrad Amman (1669-1730) und Theodoor Jannsson von Almeloveen in 4. Aufl. 1755 bei Wettstein in Amsterdam mit Anmerkungen und einem Lexicon Caelianum herausgegebenen Werks "Über die akuten und chronischen Krankheiten in acht Büchern". b Kapitelüberschrift des 1. Kapitels aus dem 5. Buch des gleichen Werks mit der Überschrift „Von den Ischias- und Lendenschmerzen" (De Ischiadicis et Psoeadicis". Es enthält die ausführlichste antike Schilderung des Ischias. Caelius unterschied noch nicht zwischen arthrogenen und neurogenen Schmerzen. (Mit freundl. Genehmigung von Dr. T. Böni)

Verlauf von mindestens einjähriger Dauer aus, der auch in seinen Symptomen deutlich schwerer verlief.

\section{》) Schon z. Zt. von Hippokrates wurde der typische Schmerz "Ischias" genannt}

Die Symptome werden ausführlich geschildert, sodass von den hippokratischen Autoren ein gutes Bild des Schmerzverlaufs abgegeben wird. Dementsprechend konnten die Beschwerden entweder nur lokal im Bereich der Lenden oder Hüfte sein oder aber auch via Leistengegend in den Unterschenkel und schließlich bis in den Fuß ziehen. Diese Symptom- und Verlaufsschilderungen des „Ischias“ darf aber retrospektiv nicht unkritisch mit der Symptomatik eines lumbalen Bandscheibenvorfalls gleichgesetzt werden, denn es wird im Zusammenhang mit dem „Ischias“ auch eine Geschwulst im Hüftbereich beschrieben, die nicht $\mathrm{zu}$ den klinischen Zeichen des lumbalem Bandscheibenvorfalls zählt. Eine eindeutige Kenntnis der Symptomatik des lumbalen Bandscheibenvorfalls darf damit den hippokratischen Autoren nicht zugeschrieben werden. Ebenso verhält es sich mit dem pathomechanistischen Verständnis; denn in den hippokratischen Schriften wird das für über Jahrhunderte gültige Krankheitskonzept der Humoralpathologie ([„Vier]-Säftelehre“) fundiert. Somit werden auch beim „Ischias“ als Krankheitsauslöser schädigende Substanzen (Flüssigkeiten), welche über die Nahrung aufgenommen und von den Patienten nicht genügend ausgeschieden werden, angenommen, wie in „Die Stellen des Menschen“ [6] dargelegt. Abhängig von der Menge kann es dann jeweils zu einer „Arthritis“ oder aber zum „Ischias“ führen.

\section{Galen von Pergamon}

Galen von Pergamon (ca. 129-200 n. Chr.), das zweite medizinische Schwergewicht der Antike, geht in der 190. Definition seiner "Medizinischen Begriffsbestimmungen“ (Definitiones medicae) ebenfalls auf den „Ischias“ ein [7]. Für Galen ist der „Ischias“ eine Art Dauerschmerz der Hüfte, welcher nicht leicht zu therapieren ist. Die Ischiasbeschwerden führen zu heftigen Schmerzen im Bereich der Hüfte, welche bis zur Kniekehle und zur Wade ausstrahlen können. Dabei betont Galen gleich den hippokratischen Autoren in seinem Werk „Über die Anordnung der Medikamente nach den Stellen" (De compositione medicamentorum secundum locos) die Verwandtschaft der Krankheiten Ischias, Arthritis und Podagra [8]. Galen war wie die hippokratischen Ärzte der Säftelehre verpflichtet und sah in schädlichen Körpersäften die Ursache für den „Ischias“.

\section{Aretaeus von Kappadokien}

Der griechische Arzt Aretaeus von Kappadokien, einer der bekanntesten Mediziner der Antike, welcher während der römischen Kaiserzeit des 1. Jahrhunderts n. Chr. gelebt hatte, berichtet ebenfalls über den „Ischias“ in seiner Schrift „Über die Ursachen und Zeichen der akuten und chronischen Krankheiten “ [9]. Dabei verwendet er den Begriff „Ischias“ in Zusammenhang mit Arthritiden und gebraucht ihn nur topologisch, sodass Aretaeus unter "Ischias“ eher ein arthritisches Hüftleiden versteht. Dem antiken humoralpathologischen Krankheitskonzept folgend, sind für Aretaeus, wie schon bei Galen erwähnt, verdorbene Säfte schuld am „Ischias“.

\section{Caelius Aurelianus}

In dem in der Spätantike entstandenen medizinischen Schriftwerk „Über die akuten und chronischen Krankheiten" (De morbis acutis et chronicis, - Abb. 1a) des römischen Arztes Caelius Aurelianus, der wahrscheinlich im 5. Jahrhundert. n. Chr. lebte, findet sich die wohl 
detaillierteste Beschreibung des „Ischias“ der Antike [10]. Es handelt sich hierbei um die lateinische Übersetzung einer verloren gegangenen griechischen medizinischen Schrift, welche dem Soranos von Ephesos (ca. 98-138 n. Chr.) zugeschrieben wird. Das 1. Kapitel des 5. Buches über die chronischen Krankheiten mit dem programmatischen Titel „Über Ischiasund Lendenschmerzen" (• Abb. 1b) ist ausschließlich dem Formenkreis „Ischias“ gewidmet. Der „Ischias“ kann in jedem Alter auftreten, gehäuft aber in den mittleren Lebensabschnitten antiker Lebenserwartung. Besonderes Gewicht legt Caelius auf die Topographie und Chronologie des Schmerzes beim Ischias, wie folgendes Zitat belegt: „... dem Wirbelschmerz folgt der Gesäßschmerz, manchmal beidseitig (doppelter Ischias), Gliederschwere, Gangschwierigkeiten ungewohnter Art, fallweise mit leichter Taubheit und Ameisenlaufen, gelegentlich mit starkem, stechendem und brennendem Schmerz und einem Gefühl des Patienten, er bewege und winde sich wie eine Schlange, die Kranken finden nicht einmal im Bett eine erträgliche Lage und zuweilen treten Fieber auf..." [10].

Im chronischen Stadium werden zudem Schwäche, Lähmungen, Atrophie und kontrakturbedingte Verkürzungen des Beins erwähnt. Trotz der detaillierten Beschreibung darf auch hier nicht vorbehaltlos die Diagnose eines lumbalen Bandscheibenvorfalls gestellt werden, da insbesondere Fieber oder Anlaufschmerzen in der Hüfte nicht dazugehören.

Im Gegensatz zu Aretaeus anerkennt Caelius neben inneren auch äußere Ursachen für die Ischiasbeschwerden. Damit ist die Ursachenliste von Caelius lang und reicht von einer „ins Mark gehenden Erkältung “ über das Liegen auf dem Erdboden bis zu „anhaltendem maßlosem Liebesgenuss“, eine Zerrung bei körperlicher Betätigung, Heben von Gewichten oder „allzu langes Verhalten des Hämorrhoidalflusses" [10].

Zusammengefasst bleibt aber der „Ischias“ von der Antike über das Mittelalter bis in die Neuzeit hinein ein heterogene Gruppe ätiologisch unterschiedlicher Erkrankungen, häufiger arthrogener als neurogener Natur, deren Ge-

Unfallchirurg 2015 - [Suppl 1]: 118:S43-S52 DOI 10.1007/s00113-015-0100-1

(c) Springer-Verlag Berlin Heidelberg 2015

\section{P. Gruber · T. Böni}

\section{Ischias. Von der Säftelehre zur Pathomorphologie}

\section{Zusammenfassung}

Heute zählt der lumbale Bandscheibenvorfall zu einem der häufigsten Krankheitsbilder, das sowohl in der Hausarztpraxis als auch in den jeweiligen Fachsprechstunden der Orthopädie, Neurologie, Rheumatologie oder Neurochirurgie gesehen wird. Zudem ist die mikrochirurgische Diskektomie einer der meist durchgeführten wirbelsäulenchirurgischen Eingriffe überhaupt. Über Jahrhunderte hinweg war die diskogene Nervenkompression ein wenig verstandenes und nicht klar abgegrenztes Krankheitsbild, für das es auch keine spezifischen und wirkungsvollen Therapien gab. Zwar haben die hippokratischen Ärzte diese Beschwerden bereits gekannt, welche sie dann auch als "Ischias" bezeichneten, aber sie subsumierten unter diesem Begriff unterschiedliche Krankheitsbilder wie z. B. Hüftbeschwerden. Es war erst das Verdienst von Domenico Felice Antonio Cotugno (1736-1822) Mitte des 18. Jahrhunderts, der begriffliche Klarheit in das

\begin{abstract}
Krankheitsbild Ischias brachte und diese Beschwerden als eine neurogen bedingte Krankheit begriff. Ebenso stammt aus dieser Zeit die erste eindeutige Beschreibung der Bandscheibe durch Josias Weitbrecht (17021747). Erst 1858 beschrieb Hubert von Luschka (1820-1875) pathoanatomisch einen Bandscheibenvorfall. Im Jahr 1908 hatten der Neurologe Oppenheim und der Chirurg Feodor Krause eine Bandscheibenoperation initiiert und erfolgreich durchgeführt, aber die Pathologie als solche verkannt. Es waren William Jason Mixter (1880-1958) und Joseph Seaton Barr (1901-1963), die 1934 die diskogen bedingte Nervenkompression als Krankheitsbild und ihre chirurgische Therapie etablierten.
\end{abstract}

Schlüsselwörter

Medizingeschichte $\cdot$ Ischias .

Bandscheibenvorfall · Anatomie .

Pathophysiologie

\section{Sciatica. From the doctrine of humors to pathomorphology}

\section{Abstract}

Lumbar disc herniation is nowadays a very common disease, which is often seen in both general practices as well as in the consultations of orthopedics, neurology, rheumatology and neurosurgery. Furthermore, lumbar disc surgery is one of the most common spinal surgical procedures worldwide; however, for many centuries, physician had no clear understanding of the anatomical condition and the pathomechanism of this condition, therefore, no rational treatment was available. The Hippocratic physicians knew the signs and symptoms of lumbar disc herniation, which they then called sciatica but subsumed different disorders, such as pelvic diseases under this term. In the mid-eighteenth century the Italian physician Domenico Felice Antonio Cotugno (1736-1822) first brought clarity into the concept of radicular syndromes. He recognized that the so-called sci- atica could be of neurogenic origin. In 1742 a contemporary of Cotugno, the German Josias Weitbrecht (1702-1747) has to be credited for the first precise description of intervertebral discs. Nearly 100 years later the German Hubert von Luschka (1820-1875) described for the first time a herniated disc in a pathological specimen. With the landmark report in the New England Journal of Medicine in 1934 the two American surgeons, William Jason Mixter (1880-1958) and Joseph Seaton Barr (1901-1963), finally clarified the pathomechanism of lumbar disc-related nerve compression and the surgical treatment.

\section{Keywords}

History of medicine - Sciatica .

Pathophysiology · Anatomy · Lumbar disc herniation meinsamkeit ein Lenden-Gefäß-BeinSchmerz war. Da ebenfalls seit den hippokratischen Schriften bis in die Mitte des 19. Jahrhunderts die Humoralpathologie die vorherrschende Lehrmeinung war, wurden als Ursache des „Ischias“ schädigende Säfte angenommen.

\section{Domenico Cotugno und der neurogene Ischias}

Erst Mitte des 18. Jahrhunderts finden sich neue medizinische Erkenntnisse zum „Ischias“. Dank den Bemühungen des italienischen Arztes Domenico Felice Antonio Cotugno (1736-1822, - Abb. 2a) 


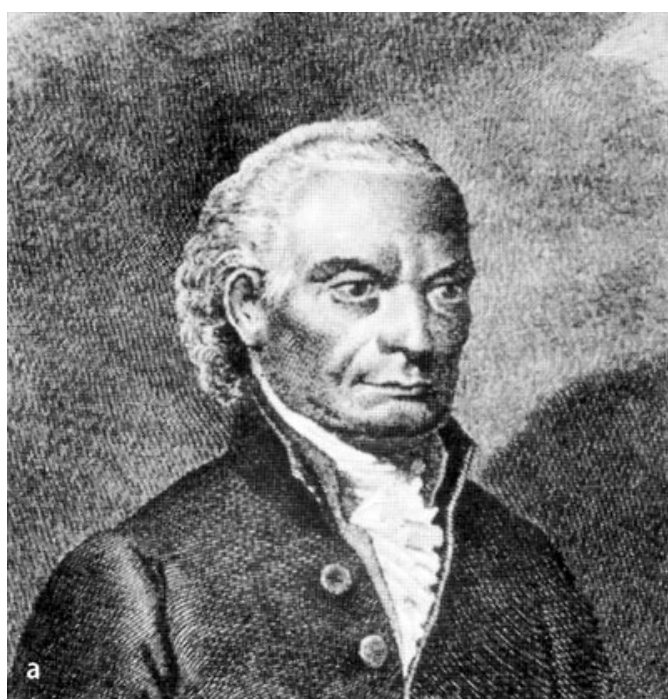

DOMINICI COTVNNII

PHIL. ET MED. DOCT.

D E

ISCHIADE NER VOSA

COMMENTARIVS.

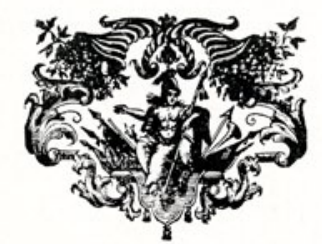

$\begin{array}{llllllllllll} & \text { N } & \text { E } & A & P & O & L & \text { I }\end{array}$ APVD FRATRES SIMONIOS MUCCLXIV.

b

Abb. $2 \Delta$ a Domenico Felice Antonio Cotugno (1736-1822) grenzte als erster das - später nach ihm Ischias nervosa Cotunni benannten - „nervige Hüftweh“ von den durch Gelenkerkrankungen, vorab arthrosebedingten Hüftschmerzen ab und leistete damit einen entscheidenden Beitrag zur Klärung des Krankheitsbildes Ischias. b Titelblatt der mit dem Druckjahr 1764 versehenen, aber erst 1765 in Neapel erschienen Erstausgabe des klassischen Werks „Kommentar über den nervösen Ischias“. Neben dem neurogenen Ischias, den er in eine „Ischias nervosa postica“ (etwa dem Dermatom L5 entsprechend) und eine "Ischias nervosa antica" (etwa den Dermatomen L3 bzw. 4 entsprechend) unterteilte, enthält das Werk auch die erste ausführliche Beschreibung des Liquor cerebrospinalis. Cotugno wurde von Albrecht von Haller (1708-1777) für seine gewissenhaften und überaus exakten Beobachtungen hoch gelobt. (Mit freundl. Genehmigung von Dr. T. Böni)

wurde ein wesentlicher Schritt zur Klärung des ätiologischen Sammeltopfs „Ischias“ gemacht, und es ist Cotugnos Verdienst, dass der „Ischias“ erstmals in den Formenkreis der Nervenkrankheiten gelangte. Dementsprechend fand sein Name Eingang in der medizinischen Terminologie mit „Ischias nervosa Cotunnii“, „Malum Cotunnii“, „Cotunnius- oder Cotugno-Syndrom “ für den neuronal bedingten Ischias.

In seiner 1764 erschienen Schrift "De ischiade nervosa commentarius" differenziert Cotugno erstmalig in der Geschichte der Medizin zwischen dem auf die Hüfte beschränkten Schmerz beim „arthritischen Ischias“ und dem durch Schmerzausstrahlung ins Bein charakterisierten "neurogenen Ischias“ (- Abb. 2b; [11]). Cotugno blieb aber nicht nur bei dieser Abgrenzung stehen, sondern teilte den neurogenen Ischias in eine "Ischias nervosa postica“ und eine "Ischias nervosa antica“ ein. Der sogenannte "posteriore Ischias" entspricht dem Verlauf nach ungefähr dem heutigen Dermatom L5, während der "anteriore Ischias“ mit dem Verlauf der heutigen dermalen Einteilung

L3 bzw. L4 korrespondiert. Als Ursache des neurogenen Ischias vermutete er eine Schwächung des Nervs durch eine scharfe und reizende Materie unklarer Herkunft. Dabei spekulierte er auch mit der Möglichkeit, dass eine Ansammlung des von ihm ebenfalls erstmals detailliert beschriebenen Liquor cerebrospinalis (Liquor Cotunnii) in der Nervenscheide dafür verantwortlich sei. Mit diesen pathophysiologischen Erklärungsversuchen blieb Cotugno aber dem Krankheitskonzept der antiken Säftelehre treu, was deutlichen Einfluss auf seine Therapievorschläge hatte und diese somit dem traditionellen Muster folgten.

\section{》) Cotugno gilt zu Recht als Erstbeschreiber des lumboradikulären Syndroms}

Zusammengefasst darf Cotugno aber dank seiner akribischen Erhebung der Krankengeschichte und Schilderung der klinischen Krankheitszeichen zu Recht als Erstbeschreiber des lumboradikulären Syndroms gelten.

\section{„Spinal irritation", eine abwegige pathophysiologische Theorie des 19. Jahrhunderts}

In der 1. Hälfte des 19. Jahrhunderts wurde die Medizin durch naturphilosophische Betrachtungen stark beeinflusst. Diesem Trend unterlagen auch die pathophysiologischen Erklärungsmodelle für die Ischiasbeschwerden. Beispielhaft hierfür ist das Konzept der „Spinal irritation“, wie diese 1840 vom deutschen Anatom und Arzt Benedikt Stilling (1810-1879), der sich u. a. mit der Beschreibung des Rückenmarks und des Kleinhirns verdient gemacht hatte, dargelegt wurde. Erstmals wurde der Begriff "spinal irritation“ vom Glasgower Arzt Thomas Brown erwähnt. Stilling widmete diesem Krankheitsbild eine ganze Abhandlung mit dem Titel „Physiologische, pathologische und medicinisch-practische Untersuchungen über die "Spinal-Irritation“ [12]. Der Rückenschmerz war das führende klinische Zeichen, aber es konnten noch viele andere Symptome hinzugefügt werden. Somit eignete sich die „spinal irritation“ gut zur Erklärung einer ganzen Reihe psychosomatischer mit Rückenschmerzen oder Ischias verbundener Krankheitsbilder. Für Stilling war die „spinal irritation“ im Wesentlichen ein Rückenmarkleiden; in einigen Fällen konnte die "spinal irritation“ aber auch durch eine Läsion eines entsprechenden Nervs ohne eigentliche Mitbeteiligung des Rückenmarks entstehen.

Die Kritik an der „spinal irritation“ blieb denn auch nicht aus. Bereits 1849 argumentierte A. Mayer mit der Schrift „Über die Unzulässigkeit der Spinalirritation als besondere Krankheit" gegen dieses Krankheitsbild [13]. Als Grund für seine ablehnende Haltung führte er an, dass für die "spinal irritation“ zu viele verschiedene Symptome angegeben worden seien, dass charakteristische Krankheitszeichen fehlen würden und dass es nicht gelungen sei, konstante Veränderungen an der Leiche nachzuweisen. Die „spinal irritation“ blieb jedoch lange Gegenstand medizinischer Diskussionen, sodass sich beispielsweise noch 1875 der bedeutende deutsche 
Internist Ernst Victor von Leyden (18321910) gezwungen sah, sich mit dieser auseinanderzusetzen [14].

\section{Moritz Heinrich Romberg}

Der deutsche Neurologe Moritz Heinrich Romberg (1795-1873) fand das Konzept der allgemeinen Nervenreizung oder Nervenschwäche für den Ischias wenig befriedigend. Dementsprechend führt Romberg in seinem "Lehrbuch der Nervenkrankheiten des Menschen" von 1840 den Rückstand in der anatomischen und diagnostischen Kenntnis des Ischias darauf zurück, dass alle Aufmerksamkeit bisher nur auf die Schenkelbahn des Hüftnervs gerichtet wurde [15]. Mit Ausnahme der selten vorkommenden Neuritis oder Verdickung und Verhärtung einzelner Nervenzweige in der Nähe von Geschwüren sei es, auch bei langer Dauer des Ischias, nie gelungen, Veränderungen von Belang im Verlauf des Nervs am Bein nachzuweisen. Man habe die Nervenläsion bisher am falschen Ort gesucht und - noch bedenklicher ein erdichteter Sektionsbefund, der eine Ansammlung seröser Flüssigkeit in den Scheiden des Ischiasnervs beschrieben habe, sollte dazu herhalten, Cotugno's mit großer Zurückhaltung geäußerte Vermutung zu bestätigen. Zur Überwindung dieses Erkenntnismangels schlägt Romberg vor, dass, wann immer sich die seltene Gelegenheit böte, die Sektion eines mit Ischias Verstorbenen vorgenommen werden solle und es dabei nicht versäumt werden dürfe, den ganzen Verlauf des Nervs bis in den Wirbelkanal einschließlich des Rückenmarks zu untersuchen. Romberg hat damit der Forschung den Weg zur Aufklärung des radikulären Kompressionssyndroms gewiesen, aber es sollten noch fast 100 Jahre vergehen, bis dieses Problem schlussendlich klinisch gelöst werden konnte.

\section{Entwicklung der klinischen Diagnostik}

Die Entwicklung spezifischer Krankheitszeichen für den Ischias entwickelte sich erst in der 2. Hälfte des 19. Jahrhunderts. Bereits 1841 beschrieb der französischen Kinderarzt François Louis Isidore Valleix
(1807-1855), welcher sich auch mit Neuralgien beschäftigt hatte und der Professor am Hôpital de la Salpêtrière in Paris war, für den neurogenen Ischias die Ischiasdruckpunkte (auch Valleix-Druckpunkte genannt [16]).

Aber erst mit den Provokationsmanövern, die vom französischen Neurologen Ernest-Charles Lasègue (18161883), welcher am Spital Necker in Paris lehrte, in seinem Beitrag „Considération sur la sciatique“ von 1864 beschrieben wurden [17], kam es zu einem signifikanten Durchbruch in der klinischen Diagnostik. Dabei unterschied Lasègue im Wesentlichen 3 Ischiaszeichen, die er auf die durch die Manöver bedingte Dehnung des Nervs zurückführte. Diese bestanden aus der Vermeidung einer gänzlichen Streckung im Hüft- und Kniegelenk bei heftigen Schmerzen, einer Schmerzprovokation durch Dorsalextension des Fußes bei initialer Plantarflexion sowie Schmerzprovokation bei Knieextension bei anfänglich angewinkeltem Kniegelenk.

\section{》) Die Provokationsmanöver von Lasègue bewirkten den Durchbruch in der klinischen Diagnostik}

Der heute weit verbreitete „LasègueTest", welcher einen Ischiasschmerz durch passives Anheben des gestreckten Beins auf der betroffenen Seite auslöst, wurde hingegen von Lasègue so nie beschrieben. Die Erstbeschreibung dieses Provokationstests verdanken wir dem serbischen Neurologen und Schriftsteller Laza K. Lazarevic (1851-1890). welche er in dem 1880 auf serbisch und in kyrillischem Alphabet erschienen Beitrag „Ischias postica Cotunnii“" schilderte [18]. Darin berichtete er von 3 Provokationstests, die alle einen Ischiasschmerz auslösen konnten und wovon das 3. Manöver dem heutigen Lasègue-Test mit Streckung des Beins auf der betroffenen Seite des in Rückenlage befindlichen Patienten äquivalent ist. Dabei hat Lazarevic jeweils die Distanz zwischen Spina iliaca posterior superior und der Ferse in gestreckter Rückenlage und bei maximalem Anheben des gestreckten Beins gemessen [19]. Die dadurch bedingte Schmerzzunahme führte er wie Lasègue auf die Dehnung des Ischiasnervs zurück.

Mit der 1881 vorgelegten Dissertationsschrift „Contribution à l'étude clinique de la sciatique" von J.J. Forst, einem Schüler von Lasègue, welche aber unabhängig von Lazarevic entstand, wurde das 3. Provokationsmanöver von Lazarevic einem breiteren Publikum bekannt [20] und dabei auf die Hohe diagnostische Wertigkeit desselben aufmerksam gemacht. Als Ursache für die Schmerzauslösung machen Forst und Lasègue den Druck der Muskeln auf den Ischiasnerv verantwortlich. Dieser angenommene Mechanismus wurde 3 Jahre später durch die Untersuchungen am Leichenexperiment durch Charles Lucien de Beurmann (1851-1923) entkräftet, indem er zeigen konnte, dass der Ischiasnerv bei der Hüftbeugung mit gestrecktem Kniegelenk stärker gedehnt wird als bei flektiertem Kniegelenk und die Muskulatur auf den Nerv keine kompressiven Kräfte auslöst [21].

In der Folge wurden zahlreiche Varianten für die Provokation des Ischiasdehnungsschmerzes entwickelt. So beschrieb der polnischen Neurologe Izydor Fajersztajn-Krzemicki (1867-1935) das in der Regel weniger stark ausgeprägte gekreuzte Ischiasphänomen [22]. Ein weiterer klinischer Provokationstest für den Ischiasdehnungsschmerz war die Dorsalextension des Fußes, welcher 1886 vom englischen Neurologen Sir William Richard Gowers (1845-1915) beschrieben wurde [23]. Ebenso haben Otto Nägeli (1843-1922 [24]) 1894, Maurice Roch (1878-1967 [25]) 1913 sowie auch Karl Bragard (1890-1973 [26]) 1928 das Lasègue-Manöver zusammen mit der Dorsalextension des Fußes, welches von Roch als „Lasègue du pied“ benannt wurde, als einen probaten klinischen Test zur Auslösung des Ischiasdehnungsschmerzes beschrieben. Zudem empfahl Vincenzo Neri (1880-1960) 1916, das Lasègue-Manöver mit einer Schmerzprovokation durch Kopfbeugen zu ergänzen, um etwaigen Simulationsversuchen vorzubeugen [27]. 


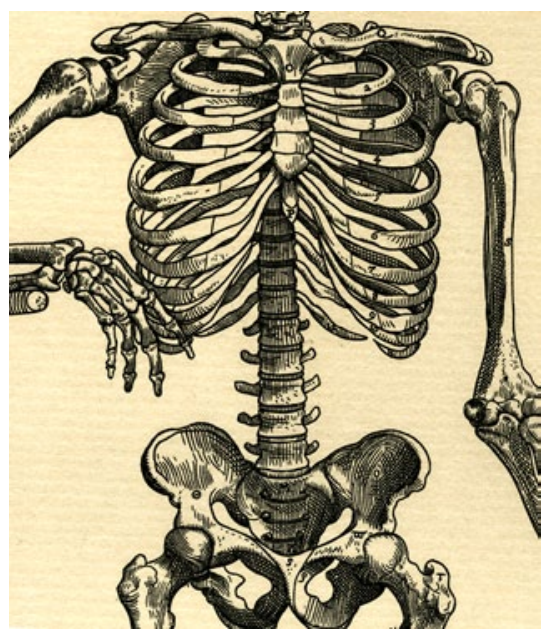

Abb. $3 \triangle$ Ausschnitt aus der ersten, dem aus den Niederlanden stammenden Maler Jan Stephan van Kalcker (ca. 1499-1546) zugeschriebenen Knochentafel für das 1543 beim Basler Drucker Johannes Oporinus (15071568) erschienene, die menschliche Anatomie revolutionierende Werk "De Humanis corporis fabrica Libri septem" von Andreas Vesal (15151584 [32]). Obwohl den Knochen gewidmet, sind auf allen einsehbaren Abschnitten der Wirbelsäule Bandscheiben erkennbar (Mit freundl. Genehmigung von Dr. T. Böni)

\section{Entwicklung der Neurotopologie des Ischias}

Nicht weniger anspruchsvoll wie die klinische Diagnostik gestaltete sich die topographische Klärung des Ischiasschmerzes. Nahm Cotugno bereits 1764 eine erste grobe Unterteilung in den vorderen und hinteren Ischias vor [11], erwies sich doch die Zuordnung der bandförmigen radikulären Schmerzen zu den peripheren Nerven als schwierig. Mit der Entwicklung der Neurochirurgie ab Mitte des 19. Jahrhunderts und dem damit verbundenen Bedürfnis nach einer exakten Neurotopographie des Gehirns wurde auch die spinale Neuroanatomie vorangetrieben. Im Jahr 1893 bot der englische Neurophysiologe und Nobelpreisträger Sir Charles Scott Sherrington (18571952) in seinem Beitrag „Experiments in examination of the peripheral distribution of the fibres of the posterior roots of some spinal nerves I" einen Überblick über den Stand der Forschung zu den Rückenmarksegmenten, den sensiblen Dermatomen [28]. Sherrington stütze dabei seine Erkenntnisse auf Tierexperimente. Im selben Jahr veröffentlichte der englische Chirurg
Sir William Thorburn (1861-1923), der ein Pionier der spinalen Chirurgie war, das bisher bekannte klinische Material [29].

Zur gleichen Zeit publizierte der englischeNeurologeSirHenryHead (18611941), nachdem auch das Phänomen der sogenannten "Head-Zonen“ benannt ist, 2 unvollständige Schemata der sensiblen menschlichen Dermatome [30], die er im Wesentlichen aufgrund des dermalen Befalls des Herpes zoster abzugrenzen lernte. Die erste komplette Darstellung der menschlichen Dermatome verdanken wir dem Schweizer Chirurgen und Nobelpreisträger Theodor Kocher (1841-1917). Er fügte sie als „Spinale Sensibilitätstafel“ und „Spinale Motilitätstafel“ seiner 1896 erschienen Monographie über die Verletzungen der menschlichen Wirbelsäule und des Rückenmarks bei [31]. Damit wurde der Weg zu einer genaueren Läsionslokalisation geebnet, der für die zukünftigen spinalchirurgischen Eingriffe wesentlich sein sollte, ohne die die ersten Bandscheibeneingriffe, wenn auch nicht als solche erkannt, erst möglich wurden.

\section{Entwicklung der Anatomie und Pathologie der Bandscheibe}

Die wohl älteste deutlich erkennbare Darstellung der menschlichen Zwischenwirbelscheiben findet sich auf Blatt 19007 verso und 19012 recto der anatomischen Zeichnungen von Leonardo da Vinci(14921519), welche in der königlichen Bibliothek auf Schloss Windsor aufbewahrt werden. Da Leonardo da Vinci seine anatomischen Beobachtungen nicht veröffentlichte, blieb diesen eine Nachwirkung in der Medizin versagt. Anders erging es aber dem 1543 erschienenen Werk „De humani corporis fabrica libri septem "des flämischen Arztes und Anatomen Andreas Vesal (15151584, Abb. 3), welches die Anatomie fundamental revolutionierte. Vesal lässt die Wirbelsäule auf allen Segmenten mit Zwischenwirbelscheiben abbilden und charakterisiert diese als knorpeliges Ligament [32]. Von der Entdeckung der Bandscheibe zur Klärung ihrer Struktur war es jedoch noch ein weiter Weg. So stellte die Bandscheibe für den einst in Leiden tätigen Pieter Paaw (1564-1617) in seinen 1615 erschienen „Primitiae anatomicae“ lediglich eine „eigenartige weißliche Masse“ (substantia pecularis albicans) dar [33]. Hundert Jahre später und zwischenzeitlich als Ligamentum intervertebrale bezeichnet, ist die Bandscheibe für den italienischen Mediziner Giovanni Battista Morgagni (1682-1771), welcher versucht hatte, sich mithilfe empirischer Untersuchungen von der gängigen Humoralpathologie zu lösen, in seinen 1719 erschienenen „Adversaria anatomica tertia“, ein Mittelding zwischen Knorpel und Band [34].

\section{》) Die Werke von Vesal revolutionierten die Anatomie}

Eine bereits tiefere Kenntnis der Bandscheibenmorphologie spricht aus der 1743 erschienenen "Exposition anatomique“ des dänischen Anatomen Jakob Benignus Winslow (1669-1760), der Professor für Anatomie am Jardin du Roi in Paris war. Für ihn ist die Bandscheibe ein Symphysenknorpel der Wirbelsäule, und er grenzt die äußeren Schichten als knorpelige Ringe ("cerceaux cartilagineux") vom weiter innen gelegenen, aus einer viel weicheren Masse bestehenden Zentrum ab [35]. Die Räume zwischen den Knorpelringen seien mit einem schleimigen Saft gefüllt, der jedoch zähflüssiger sei als die Synovia [35]. Die vortrefflichste Beschreibung der Bandscheibe im 18. Jahrhundert lieferte Josias Weitbrecht (1702-1747), der als Professor für Physiologie und Anatomie in St. Petersburg wirkte und als einer der bedeutendsten Anatomen seiner Zeit galt. In seiner 1742 erschienenen "Syndesmologia“ (• Abb.4a) untersuchte er die Richtung der Fasern des Anulus fibrosus und beobachtete ihre schräg aufsteigenden Bündel sowie den sich entgegengesetzt kreuzenden Verlauf der verschiedenen aus ihnen gebildeten Lamellen mit einem zentral gelegenen gallertartigen Kern (- Abb. 4b; [36]). Anfang des 19. Jahrhunderts wurde vom französischen Anatom und Arzt Baron Antoine Portal (1742-1832), welcher Professor für Anatomie am Jardin du Roi in Paris und Leibarzt von Louis XVIII. und Charles X. war, in seinem 1804 erschienenen 5-bändigen Werk „Cours d'anatomie médicale“, die Bandscheibe als eine kompartimentierte anatomische Struktur, die aus einer lamellenfreien zentralen Höhle mit einer 


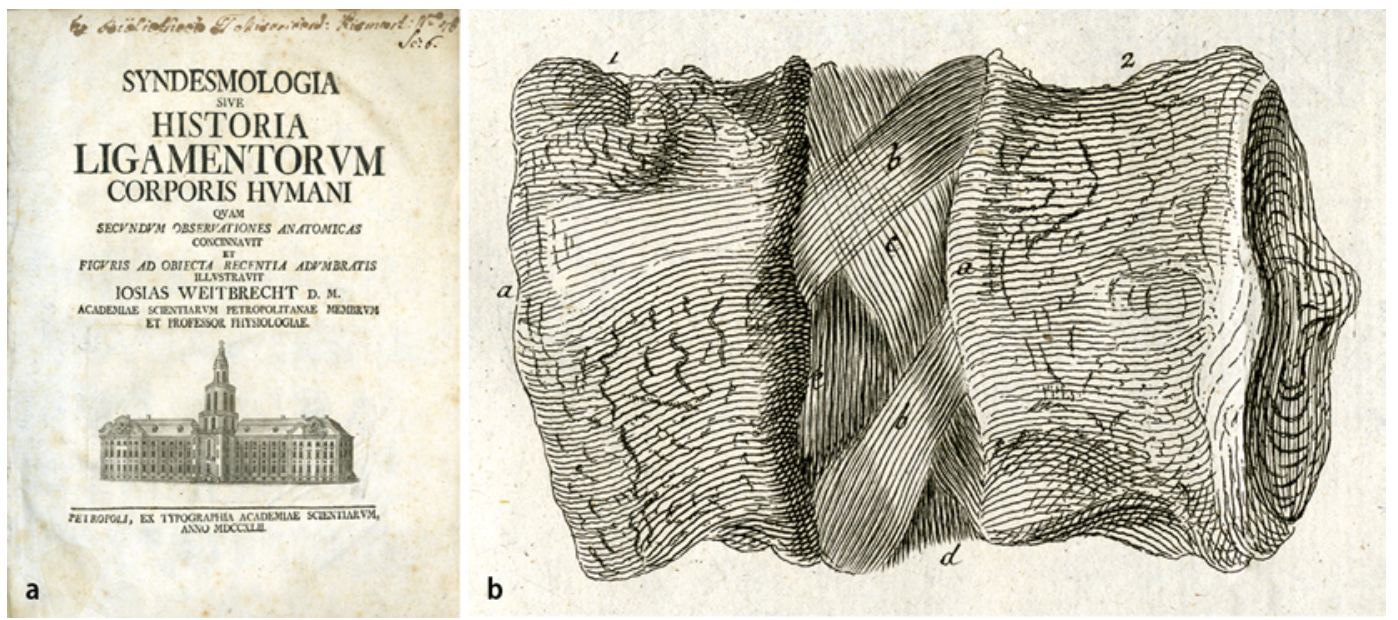

Abb. $4 \Delta$ a Titelblatt der 1742 in Petersburg erschienenen „Syndesmologia“ von Josias Weitbrecht (1702-1747), seinem bis heute durch den Detailreichtum und die hervorragenden Illustrationen beeindruckenden Meisterwerk über die Knochenverbindungen [36]. Das dargestellte Gebäude, damals Sitz der Petersburger Akademie der Wissenschaften, beherbergt heute die Petersburger Kunstkammer. b Fig. 42 der Tafel XII zeigt deutlich die schräg aufsteigende Richtung der Faserbündel des Anulus fibrosus und den sich entgegengesetzt kreuzenden Verlauf der verschiedenen aus ihnen gebildeten Lamellen [36]. (Mit freundl. Genehmigung von Dr. T. Böni)

heterogenen, schleimigen Masse bestand, beschrieben [37].

\section{Hubert von Luschka und die Erstbeschreibung des Bandscheibenvorfalls}

Der deutsche Anatom Hubert von Luschka(1820-1875), welcheralsProfessor für Anatomie an der Universität Tübingen tätig war, wies die Beständigkeit einer Höhle in allen normal beschaffenen Bandscheiben der beweglichen Wirbelsäule in seiner 1858 erschienen Monographie „Die Halbgelenke des menschlichen Körpers" nach (- Abb. 5a; [38]). Dies bewog ihn, die sogenannten Wirbelsynchondrosen des Menschen auf Gelenke zurückzuführen. Dementsprechend interpretierte er den Anulus fibrosus als Gelenkkapsel, die überknorpelten Wirbelendflächen als eigentliche Gelenkflächen und den Gallertkern als Synovialhaut. Erstmalig in der Medizingeschichte blieb Luschka jedoch nicht nur bei der Beschreibung der normalen Anatomie stehen, sondern ging auf deren pathologische Veränderungen ein. Grundlage für sein Verständnis der Bandscheibenerkrankungen war für ihn wiederum die von ihm postulierte Verwandtschaft mit den vollständigen Gelenken. Als typisches Beispiel dafür führte er die chronische, mit geringeren oder größeren Veränderungen einher- gehende Entzündung derselben an, die überraschende Ähnlichkeiten mit dem Malum senile (Arthrose) der anderen Gelenke besitze.

Seine Schilderung der zahlreichen von ihm beobachteten krankhaften Veränderungen der Bandscheibe beschließt Luschka mit der Erstbeschreibung eines Bandscheibenvorfalls. Als sehr beachtenswerte Anomalien an den Wirbelkörperverbindungen fielen ihm knorpelige Auswüchse auf. Er unterschied 2 Lokalisationen: einerseits ventral und lateral, andererseits dorsal. Dem dorsalen Knorpelauswuchs, den er 2-mal beobachten konnte und der seines Wissens noch nie beschrieben worden war, maß er eine große praktische Bedeutung zu. Er brachte sie mit der von ihm und Virchow entdeckten Knorpelgeschwulst am Clivus Blumenbachii in Verbindung. Im ersten Fall beschrieb er den Knorpelauswuchs an der Mitte der hinteren Seite des Faserrings zwischen dem 12. Brust- und dem 1. Lendenwirbel bei einem 45 Jahre alten, im zweiten Fall zwischen dem 2. und 3. Lendenwirbel bei einem 36-jährigen Mann. Beide Männer waren an akuten Krankheiten verstorben und wiesen keine anderen Anomalien an der Wirbelsäule auf. Sehr anschaulich beschrieb er die rundlichen, kaum den Umfang einer kleinen Bohne erreichenden, gelappten, gallertartig weichen und gräu- lich gefärbten Knorpelauswüchse, welche über den Anulus fibrosus in den Wirbelkanal hineinragten. Da das hintere Längsband keinerlei Veränderungen aufwies, wäre ihm diese Entdeckung beinahe entgangen. Nur weil er die Beziehung des Anulus fibrosus zum hinteren Längsband erforschen wollte, löste er das letztere vorsichtig ab. Darunter fand er die Auswüchse, deren Vordringen stellenweise bereits zu einer Verdünnung des hinteren Längsbandes geführt hatten. Luschka erkannte sofort die klinische Bedeutung seiner Beobachtungen und zweifelte nicht daran, dass bei fortschreitendem Wachstum dieser Auswüchse das hintere Längsband durchbrochen und das Rückenmark komprimiert würde. Die mikroskopische Untersuchung ergab eine teils fibrilläre, teils strukturlose Bindesubstanz mit eingelagerten Knorpelzellen. Als Ursprung dieser gallertig-knorpeligen Auswüchse konnte er ohne Schwierigkeiten den Nucleus pulposus identifizieren (• Abb. 5b, c; [38]).

\section{》) H.v. Luschka war der Erstbeschreiber des Bandscheibenvorfalls}

Luschka schrieb in seiner Abhandlung stets von „Auswuchs" und "Geschwulstmasse“, das klar zeigt, dass er diese 

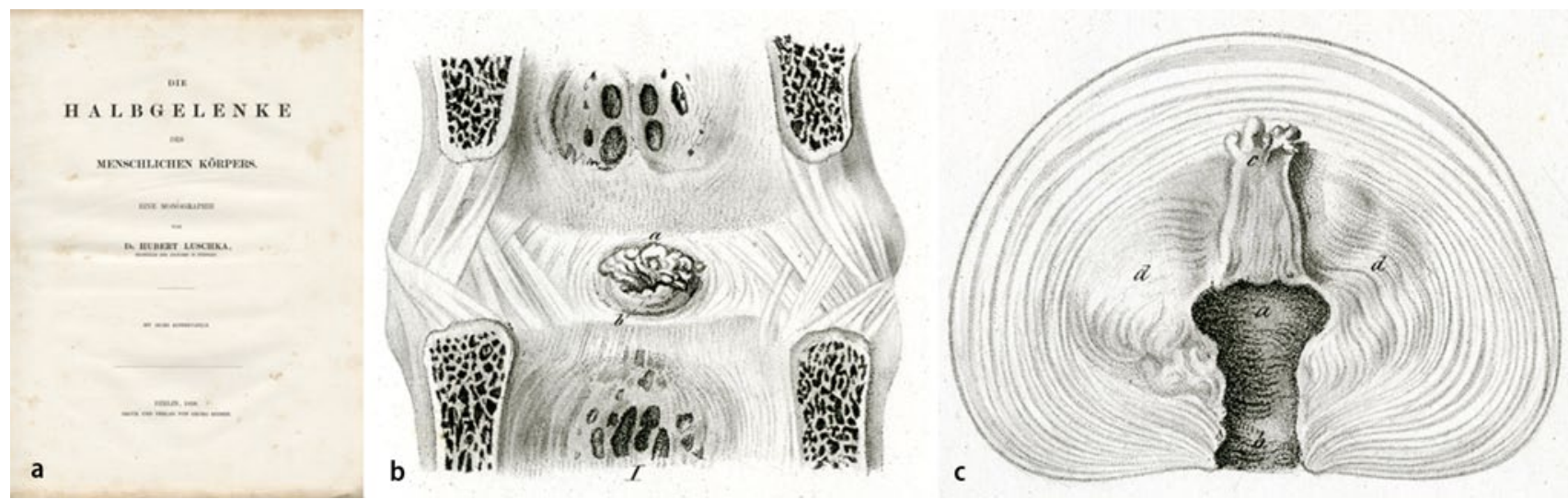

Abb. $5 \Delta$ a Titelblatt von Hubert von Luschkas (1820-1875) Monographie über die menschlichen Halbgelenke, in der er die sogenannten Wirbelsynchondrosen wegen der im Inneren der Bandscheiben beobachteten Höhle auf den Typus der hohlen Knochenverbindungen, d. h. der Gelenke zurückführt. Das Werk enthält die erste bisher bekannte Beschreibung eines Bandscheibenvorfalls nach hinten. Er interpretierte den Bandscheibenvorfall als Geschwulst bzw. Auswuchs, d. h. als Neubildung [38]. b Fig. 8 aus Tafel II zeigt die Ansicht von hinten auf die Bandscheibe Th12/L1:„An der Mitte der letzteren kommt eine fein gelappte, ein rundliches, weiches Geschwülstchen a. darstellende Masse zum Vorscheine, welche der Faserring bei b. durchbrochen hat und unter das Lig. post. zu liegen kam" [38]. c Fig. 9, Tafel II zeigt einen Transversalschnitt durch die gleiche Bandscheibe: „Es ist eine sehr umfängliche Höhle a. bemerklich, welche sich nach hinten zu einem Kanale b. verlängert, der in der Mitte der hinteren Seite der Knorpelscheibe ausmündet. Durch ihn ist eine Fortsetzung c. des Gallertkernes d. in den Wirbelkanal unter das Lig. longitud. postic. hereingewuchert“ [38]. (Mit freundl. Genehmigung von Dr. T. Böni)

Struktur für eine Neubildung hielt. Von einer Ruptur des Anulus fibrosus, einer Verlagerung oder gar einem Vorfall des Nucleus pulposus spricht er nicht. Dies führte wohl auch dazu, dass auch nachfolgende Autoren, die sich mit dem diskogenen Nervenkompressionssyndrom beschäftigten, wie beispielsweise die deutschen Mediziner Heinrich O. Oppenheim (1858-1919) und Fedor Krause (1857-1937 [39]) oder auch der Schweizer Neurologe Otto Veraguth (1870-1944 [40]) primär von Neubildungen ausgingen.

Zusammenfassend kann festgehalten werden, dass Luschka ein subligamentäres-intraligamentäres Auswachsen der knorpelig-gallertigen Masse aus dem Nucleus pulposus beschrieben und einen Durchbruch durch das hintere Längsband mit Beeinträchtigung des Rückenmarks vorweggenommen hat.

\section{Von Traumata und Knorpelknötchen}

Der Schweizer Chirurg Theodor Kocher (1841-1917), der sich wie schon erwähnt, um die Neurotopologie mit der exakten dermalen Zuordnung verdient gemacht hatte, schrieb 1896 über einen traumatisch bedingten Bandscheibenvorfall in $\mathrm{Zu}$ sammenhang mit einer kompletten Bandscheibenzertrümmerung zwischen den Wirbelkörpern L1 und L2 nach Sturzereignis eines 26-jährigen Mannes aus ca. $30 \mathrm{~m}$ Höhe [31]. Aufgrund der Ausführungen von Kocher kann aber nicht davon ausgegangen werden, dass er eine diskogen bedingte Schädigung der neuronalen Strukturen des Spinalkanals annahm. Fünfzehn Jahre später berichteten die beiden englischen Mediziner George S. Middleton (1853-1928) und John H. Teacher (1869-1930) in der Glasgower medizinischen Zeitschrift über einen Industriearbeiter, der nach einem Arbeitsunfall durch das Anheben einer schweren Eisenplatte eine Paraplegie entwickelt hatte. Da der Patient das Ereignis nur um 16 Tage überlebte, führten Middleton und Teacher eine Autopsie durch, bei welcher sich prolabiertes Bandscheibengewebe als Ursache für die neurologischen Ausfälle herausstellte [41]. Damit postulierten Middleton und Teacher, dass Traumata als Mechanismus für Bandscheibenvorfälle ursächlich sein könnten. Diese These versuchten sie experimentell zu erhärten.

Ein weiterer Erklärungsversuch für diese kartilaginären Tumoren im Spinalkanal wurde von den deutschen Pathologen Christian Georg Schmorl (18611932) und seinem Assistenten Rudolf Andrae, welche zusammen am pathologischen Institut des Stadtkranken- haus Dresden-Friedrichstadt gearbeitet haben, abgegeben. Auf Schmorl geht nicht nur der nach ihm benannte Bandscheibenvorfall in die Wirbelkörperspongiosa, der sogenannte „Prolapsus disci intraspongiosus" oder "Nodulus Schmorl“, zurück, sondern er beschrieb 1928 in „Über Chordareste in den Wirbelkörpern" auch 2 Fälle mit dorsal der Bandscheibe gelegenen halbkugeligen Vorwölbungen in den Wirbelkanal. Er interpretierte diese als akzessorische Nuclei pulposi, d. h. als Chordareste [42].

Andrae ist diesen Befunden in seinem 1929 erschienen Beitrag „Über die Knorpelknötchen am hinteren Ende der Wirbelbandscheiben im Bereich des Spinalkanals" nachgegangen [43]. Er konnte dabei die Befunde von Schmorl bei der Untersuchung von 368 Wirbelsäulen in 15,2 \% der Fälle bestätigen. Diese Knorpelknötchen fanden sich ausschließlich bei über 30-jährigen Individuen im Bereich der mittleren und unteren Brustwirbel- sowie an der oberen Lendenwirbelsäule. Eine Rückenmarkkompression durch die etwa zur Hälfte mehrfach vorkommenden Knorpelknötchen konnte aber nicht beobachtet werden. Für die Entstehung dieser Knorpelknötchen zog er 2 Mechanismen in Betracht. Durch die Zerreißung der Knorpelplatte in der Wirbelmitte entsteht ein Knorpelknoten, 
Tab. 1 Konzeptgeschichte des Bandscheibenvorfalls im Zeitstrahl

\begin{tabular}{|ll}
\hline Zeit & Antomie, Pathophysiologie, Diagnostik \\
\hline 1550 v. Chr. & Im Papyrus Edwin Smith früher Bericht von Rückenbeschwerden \\
\hline 5. Jhdt. v. Chr. & Einführung des Begriffs Ischias durch die hippokratischen Ärzte \\
\hline 1543 & Frühe Bandscheibendarstellung durch Vesal \\
\hline 1742 & Weitbrecht stellt die Bandscheibe korrekt dar \\
\hline 1764 & Cotugno unterscheidet den arthrogenen vom neurogenen Ischias \\
\hline 1858 & Luschka beschreibt zum ersten Mal einen Bandscheibenvorfall \\
\hline 1881 & Forst beschreibt das Lasègue-Zeichen \\
\hline 1896 & Kocher führt die Dermatome ein \\
\hline 1929 & Andrae klärt die Pathologie der Diskusprotrusion \\
\hline 1934 & Mixter und Barr verhelfen zum Durchbruch des diskogenen \\
& Kompressionssyndroms als Krankheit \\
\hline
\end{tabular}

der unter Druckeinwirkung zusammen mit Gewebemassen aus dem Nucleus pulposus wegen des stärkeren Widerstands der Knochenlamellen der Bandscheibe entlang bis an die Hinterfläche des Wirbels gelangt und dort, „vielleicht durch eine Art von Druckatrophie", allmählich den Anulus fibrosus zerstört. Diesen Entstehungsmechanismus hielt er selten für zutreffend. Viel häufiger zog er einen zweiten Entstehungsmechanismus in Betracht, bei dem der Nucleus pulposus sich bei intakten oder nur wenig beschädigten Knorpelplatten innerhalb der Bandscheibe einen Weg nach hinten sucht, was natürlich nur bei einer Zerstörung des Anulus fibrosus möglich ist. „Auf diesem Wege ist also das Nucleusgewebe, das sich in Degeneration befand, durch die wohl ebenfalls infolge beginnender Degeneration geschädigten und zerstörten Fasern des Anulus fibrosus zunächst bis zum Lig. Long. Post. vorgedrungen".

Ganz in Analogie zu Schmorl's Knötchen werden auch diese Knorpelknötchen als Prolaps bzw. Herniation von Bandscheibengewebe aufgefasst. Ob ein Trauma für die Knorpelknötchen verantwortlich ist oder gar ein rein degenerativer Prozess ausreicht, vermochte Andrae nicht zu entscheiden. Dass aus den Knorpelknötchen Neubildungen wie ein Chondrom oder Chordom entstehen würden, konnte er nie beobachten. Andraes Untersuchungen trugen Entscheidendes zur Klärung des Krankheitsgeschehens bei, vermochten jedoch keinen Zusammenhang mit dem Ischias herzustellen.
Es waren schlussendlich erst die beiden amerikanischen Chirurgen William Jason Mixter (1880-1958) und Jospeh Seaton Barr (1901-1963), die mit der Fallserie von 25 Patienten, welche 1934 im New England Journal of Medicine publiziert wurde [1], den Nachweis erbrachten, dass der Bandscheibenvorfall als Ursache für den Ischias zu werten ist. Damit eröffneten Mixter und Barr auch neue Wege der Therapie und verhalfen dem chirurgischen Eingriff bei der Behandlung des neurogenen Kompressionssyndroms zum Durchbruch.

\section{Der Bandscheibenvorfall im Lauf der Geschichte}

\section{Hierzu s. • Tab. 1.}

\section{Fazit für die Praxis}

- Die hippokratischen Ärzte führten den Begriff „Ischias“ für eine ganze Bandbreite verschiedener Krankheiten mit ähnlichen Symptomen ein. Eine klinische Unterscheidung zwischen einem arthrogen oder neurogen bedingten Ischias konnten sie nicht vornehmen. Dementsprechend existierte auch keine kausale Therapie für diese Beschwerden während der gesamten Antike.

- Erst Domenico Felice Antonio Cotugno (1736-1822) führte 1764 mit seiner Arbeit über den Ischias die klinische Differenzierung zwischen einem „arthrogenen“ und „neurogenen“ Ischias ein und unterschied zudem zwischen einem anterioren und posterioren neurogenen Ischias.

- Dank der Arbeiten von Sir Charles Scott Sherrington (1857-1952) und später Theodor Kocher (1841-1917) mit der Beschreibung der Dermatome konnte eine exakte neurotopologische Diagnostik erfolgen.

- Obwohl sich bei Leonardo da Vinci (1492-1519) und Andreas Vesal (1515-1584) erste Darstellungen der Bandscheibe finden, wurde die Bandscheibe erst durch Josias Weitbrecht (1702-1747) 1742 anatomisch korrekt dargestellt.

- Dass die Bandscheibe auch pathologische Veränderungen haben kann, wurde erstmals 1858 von Hubert von Luschka (1820-1875) beschrieben. Die Einordnung dieser Veränderungen als einfacher Bandscheibenvorfall blieb bis zu den Arbeiten von Rudolf Andrae in den 30 er Jahren des 20. Jahrhunderts verborgen.

- Erst durch die Arbeit von William Jason Mixter (1880-1958) und Joseph Seaton Barr (1901-1963), welche 1934 im New England Journal of Medicine erschien, wurde der Zusammenhang zwischen einem Bandscheibenvorfall mit konsekutiver Kompression der Spinalwurzel und dem Ischias gestellt.

\section{Korrespondenzadresse}

\section{Dr. P. Gruber}

Klinik für Neurologie

Universitätsspital Zürich

Frauenklinikstrasse 26, 8091 Zürich

philipp.gruber@usz.ch

\section{Einhaltung ethischer Richtlinien}

Interessenkonflikt. P. Gruber und T. Böni geben an, dass kein Interessenkonflikt besteht.

Dieser Beitrag beinhaltet keine Studien an Menschen oder Tieren.

This article is part of a supplement sponsored by SIGNUS Medizintechnik GmbH.

\section{Literatur}

1. Mixter WJ, Barr JS (1934) Rupture of the intervertebral disc with involvement of the spinal canal. N Engl J Med 211:210-215 
2. Boeni T (2000) Vom Ischias zum Bandscheibenvorfall - Zur Geschichte eines Krankheitskonzeptes. Orthopäde 29:1033-1043

3. Gruber P, Boeni T (2008) History of spinal disorders. In: Boos N, Aebi M (Hrsg) Spinal Disorders Fundamentals of diagnosis and treatment. Springer, Berlin

4. WestendorfW (1966) Papyrus Edwin Smith: Ein medizinisches Lehrbuch aus dem Alten Ägypten. Hubers Klassiker der Medizin und der Naturwissenschaften, Bd IX. Hans Huber, Bern

5. Scurlock JA, Andersen BR (2005) Diagnosis in Assyrian and Babylonian Medicine. University of Illinois, USA

6. Hippokrates (1895-1900) Sämmtliche Werke. Ins Deutsche übersetzt und ausführlich commentiert von Dr. R. Fuchs, Bd I-III. Lüneberg, München

7. Galen von Pergamon (1830) Definitiones medicae. Claudii Galeni opera omnia. In: Kühn DCG (Hrsg) Medicorum Graecorum opera quae exstant, vol XIX. Officina Car. Cnoblochii, Leipzig

8. Galen von Pergamon (1827) De compositione medicamentorum. Claudii Galeni opera omnia. In: Kühn DCG (Hrsg) Medicorum Graecorum opera quae exstant, vol XIII. Officina Car. Cnoblochii, Leipzig

9. Allan DB, Waddell G (1989) An historical perspective on low back pain and disability. Acta Orthop Scand Suppl 60:3

10. Caelius A (1755) De Ischiadicis et Psoeadicis, Morborum Chronicorum, liber V. In: Ammann JC, von Almeloveen TJ (Hrsg) Caelii Aureliani Siccensis, De Morbis Acutis et Chronicis. Freie Übertragung von B. Rüttimann. Wettstein, Amsterdam, S $547 f$

11. Cotunnius $D$ (1764) De ischiade nervosa commentarius. Typographia Simoniana, Neapel

12. Stilling B (1840) Physiologische, pathologische und medicinisch-practische Untersuchungen über die Spinal-Irritation. Wigand, Leipzig

13. Mayer A (1849) Ueber die Unzulässigkeit der Spinalirritation als besondere Krankheit. Zabern, Mainz

14. Leyden von E (1875) Klinik der Rückenmarkskrankheiten. Hirschwald, Berlin

15. Romberg MH (1840) Lehrbuch der Nervenkrankheiten des Menschen. Duncker, Berlin

16. Valleix FLI (1841) Traité des névralgies,ou affections douloureuses des nerfs. Deutsche Übersetzung: Abhandlung über die Neuralgien (1852) Vieweg, Braunschweig

17. Lasègue CE (1864) Considérations sur la sciatique. Arch Gén Méd 2(7):558-580

18. Lazarevic LK (1880) Ischias postica Cotunnii. Archivum Serbicum pro universa scientia et arte medica recipienda 7:23-25

19. Karbowski K, Radanov BP (1995) The history of the discovery of the sciatica stretching penomenon. Spine 13:1315-1317

20. Forst JJ (1881) Contribution ã l'étude clinique de la sciatique. Thèse No 33 Faculté de Médecine, Paris

21. Beurmann de $L$ (1884) Note sur un signe peu connu de la sciatique: Recherches expérimentales. Arch Phys Norm Pathol 16:375-380

22. Fajersztajn-Krzemicki I (1901) Ueber das gekreuzte Ischiasphänomen. Ein Beitrag zur Symptomatologie der Ischias. Wien Klin Wochenschr 14:41-47

23. Gowers WR (1886) A manual of diseases of the nervous system. Churchill, London

24. Naegeli O (1894) Therapie von Neuralgien und Neurosen. Sallmann, Basel

25. Roch M (1913) Lasègue du pied. Rev Med Suisse Rom 33:500-501
26. Bragard K (1928) Über das Lasèguesche Phänomen. Münchner Med Wochenschr 75:387389

27. Neri V (1916) Per scoprire la simulazione della sciatica. Quaderni Psychiatr 3:107-108

28. Sherrington CS (1893) Experiments in examination of the peripheral distribution of the fibres of the posterior roots of some spinal nerves I. Philos Trans R Soc 184B:641-763

29. Thorburn W (1893) The sensory distribution of the spinal nerves. Brain 22:355-374

30. Head H (1893) On disturbances of sensation with special reference to the pain of visceral disease. Brain 16:1-132

31. Kocher T (1896) Die Verletzungen der Wirbelsäule zugleich als Beitrag zur Physiologie des Rückenmarks, II. Die Läsionen des Rückenmarks bei Verletzungen der Wirbelsäule. Mitt Grenz Geb Med Chir 1:415-660

32. Vesal A (1543) De Humani corporis frabrica Libri septem. Froben, Basel

33. Pauw $P$ (1615) Primitiae anatomicae. De humani corporis ossibus. Colster, Leyden

34. Morgagni GB (1719) Adversaria anatomica tertia. Cominus, Padua

35. Winslow JB (1743) Exposition anatomique de la structure du corps humain. Tourneisen, Amsterdam

36. Weitbrecht J (1742) Syndesmologia, sive historia ligamentorum corporis humani. Typ Acad Sci, Petersburg

37. Portal A (1804) Cour d'anatomie médicale, ou élémens de l'anatomie de l'homme. Baudouin, Paris

38. Luschka H (1858) Die Halbgelenke des menschlichen Körpers. Eine Monographie. Reimer, Berlin

39. Oppenheim H, Krause F (1909) Ueber Einklemmung bzw. Strangulation der Cauda equina. Dtsch Med Wochenschr 35:697-700

40. Veraguth O (1929) Neurologische Skizzen. Schweiz Med Wochenschr 10:154-158

41. Middleton GS, Teacher JH (1911) Injury to the spinal cord due to rupture of an intervertebral disk during muscular effort. Glasgow Med J 76:1-6

42. Schmorl G (1928) Ueber Chordareste in den Wirbelkörpern. Zentralbl Chir 37:2305

43. Andrae R (1929) Ueber Knorpelknötchen am hinteren Ende der Wirbelbandscheiben im Bereich des Spinalkanals. Beitr Pathol Anat 82:464-474 